\title{
Some functional characteristics of the oesophageal vestibule in infants and children
}

\author{
A. R. CHR ISPIN, G. W. FRIEDLAND, A ND D. E. WR I G H T \\ From the Department of Diagnostic Radiology, The Hospital for Sick Children, Great Ormond \\ Street, London, W.C.1, and the Department of Civil Engineering, Imperial College of Science and \\ Technology, London, S.W.7
}

The oesophageal vestibule lies immediately above the stomach and during quiet respiration is located partly in the thorax, partly in the hiatus in the diaphragm, and partly in the abdomen. An analysis of some aspects of function of the vestibule in infants and children who have a noncontractile gullet above the vestibule is presented. Opening of the vestibule is related to deglutition and is independent of the oesophagus above. In its opening and closing the vestibule is shown to have the characteristics of a 'muscle throttle'. The rapidity of the event of either opening or closing is accounted for. The important relationship of calibre of lumen of the vestibule to flow through it is analysed. The effect of the indentations of mucosa into the lumen when the vestibule is partially open is analysed from a theoretical standpoint, and it is inferred that the vestibule when only partially open has the characteristics of a highly efficient choke.

The vestibule lies at the lower end of the oesophagus immediately proximal to the stomach. Normally, part of the vestibule lies in the abdomen, part in the diaphragmatic hiatus, and a part, during quiet respiration, lies in the thorax. In the normal infant or child radiological studies of the function of the vestibule are handicapped by the direct effects of deglutition and the presence of an oesophageal stripping wave emptying the oesophagus of its content after deglutition. Paediatric radiological practice provides the opportunity of assessing vestibular function in the absence of a stripping wave and also when the direct effect of deglutition can be excluded.

\section{PATIENTS STUDIED}

Four groups have been included in the present study: Group 1 Tracheo-oesophageal fistula: Two patients with an absence of oesophageal stripping action who had had no operative treatment.

Group 2 Post-repair of oesophageal atresia: Four patients were examined, none of whom showed an oesophageal stripping wave. In effect, this repair is a repair of an ossophageal transection. In three of the four a small fixed sliding type hiatal hernia was present.

Group 3 Colon transplantation for oesophageal atresia with the transplant interposed between short upper and lower oesophageal segments: Six patients were examined; no mass movements were observed in the transplant at the time of the examination.

Group 4 Colon transplant with a lower anastomosis between the lower oesophageal segment and the transplant: Proximally the colon had been exteriorized on the left side of the neck and there was a right upper oesophagostomy. In this one patient who was $\frac{}{\Phi}$ being treated for oesophageal atresia there was no continuity of lumen.

By giving a barium mixture and then positioning the patient the effect of the act of deglutition was observed. The children were quiet during the examination. Sobbing and crying, associated as they are with acts of deglutition, make a satisfactory assessment impossible.

\section{OBSERVATIONS AND DEDUCTIONS}

The vestibule remained closed except after deglutition. The timed interval between the act of

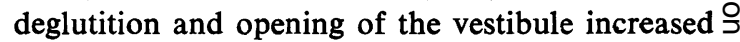
with increasing age. Thus it was shown that open- $\rightarrow$ ing and closing of the vestibule are not related to the stripping wave in the oesophagus, oesophageal $N$ continuity, or to continuity of the lumen between the pharynx and the lower oesophagus. It can be ${ }_{N}$ deduced that the opening and closing of the $\omega$ vestibule are dependent on a neural pathway? independent of the oesophagus (Chrispin ande Friedland, 1966).

Normally at the level of the diaphragm there is $\stackrel{?}{+}$ a 'point of reversal' between the negative intra- $\frac{T}{\circ}$ thoracic pressure and the positive intra-abdominal $\underset{\mathbb{D}}{\stackrel{D}{*}}$ pressure. It has been possible to exclude the effect $\stackrel{\mathcal{Q}}{\mathcal{P}}$ of this pressure difference on the opening and $\AA$ closing of the vestibule in these patients and in $\overline{0}$ particular in certain instances: (a) intraluminally, 
in the patient with the interrupted gullet by having a tube open to the atmosphere in the proximal end of the colon transplant and an open gastrostomy tube $;(b)$ intraluminally, in one patient in group 3 who showed bidirectional flow with contrast medium passing distally into the stomach and air passing proximally from the stomach into the transplant through the open vestibule; and (c) extraluminally, in three patients in group 2 who had a small but fixed type of hiatal hernia with the vestibule located in the thorax. In one patient in group 3 the patient was asked to make a dry swallow, and this was followed by opening and then closing of the vestibule.

EFFECT OF OPENING AND CLOSING OF THE VESTIBULE ON FLOW When fluids only are considered Poiseuille's equation is interesting and a physical analogy useful. The equation may be stated in the form

$$
\mathrm{Q}=\frac{\Delta \mathrm{p}}{\Delta 1} \frac{\pi \mathrm{r}^{4}}{8 \mu}
$$

where $Q$ is the flow of a fluid of viscosity $\mu$ through a cylindrical tube of radius $r$ and length $\Delta 1$ where there is a difference of pressure along the tube of $\Delta p$ (Fig. 1).

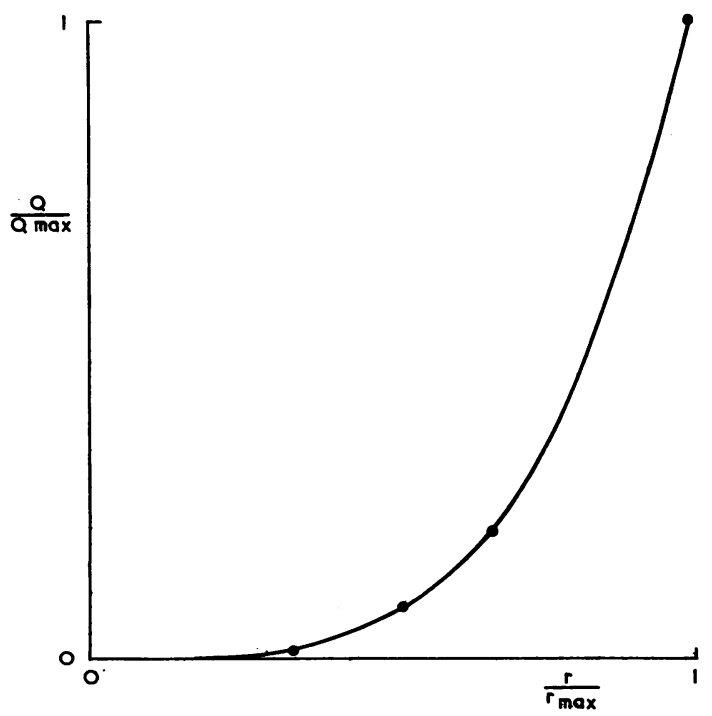

FIG. 1. Poiseuille equation $Q=\frac{\triangle p-r^{4}}{\triangle I 8 \mu}=K r^{4}$. Graph of $\frac{Q}{Q \max }=\left(\frac{r}{r \max }\right)^{4}$ against $\frac{r}{r \max }$ where $Q$ is flow at radius $r$ and $Q$ max is the maximum flow at maximum radius $r$ max. This demonstrates the influence of change of ralius on flow.
The viscosity of the contrast medium does not change. In these patients, from moment to moment, the length of the vestibule and pressure gradient across it change little, but the calibre of the vestibule changes considerably. In the Poiseuille equation the effect of a change in calibre is raised to the fourth power. Thus, in general terms, small changes in the calibre of the vestibule result in large changes in flow through it.

IMPORTANCE OF THE MUCOSA IN RELATIONSHIP TO THE MUSCLE The mucosa of the vestibule is encircled by a layer of muscle. It is unlikely that the total cross-sectional area of the mucosa changes very much during opening and closing of the vestibule. When the vestibule is closed the mucosa is folded within the cuff of muscle. When the vestibule opens fully these folds are no longer evident and the mucosa forms a thin inner layer lining the muscle ; in this way elongation of muscle gives an increase in the cross-sectional area of the lumen. The combination of a muscle layer lined by a layer of mucosa makes it possible to close the lumen of the vestibule and also to provide a wide lumen as the muscle increases its circumference.

When closed the vestibule has linear streaks of contrast medium lying between the folds of mucosa. Folding of the mucosa produces indentations into the lumen giving a roughly stellate appearance on cross-section. Changes in the crosssectional characteristic of the lumen of the vestibule affect flow through it. The resultant alteration in hydraulic capacity can now be considered (Fig. 2). A coefficient $\alpha$ is introduced into the equation so that

$$
\begin{gathered}
\mathrm{Q}=\alpha \mathrm{Qp} \\
\text { where } \mathrm{Qp}=\frac{\Delta \mathrm{p} \pi \mathrm{r}_{\mathrm{e}}^{4}}{\Delta 18 \mu} \\
\text { and } \mathrm{Q} \text { is the actual flow. }
\end{gathered}
$$

$\alpha$ is a dimensionless coefficient dependent upon the ratio of the cross-sectional area of the 'lumen' to the perimeter of the 'lumen', lumen being the effective flow conduit. It can be seen that the less the lumen is like a cylinder the less is its hydraulic capacity. This means that when the vestibule is incompletely open flow is very much less than it would be if one were considering a cylinder of circular cross-section and radius $r_{\mathrm{e}}$. Thus, the vestibule has the characteristics of a highly efficient choke when it is incompletely open because of the folding of the mucosa. As the vestibule opens fully the mucosal folds are 


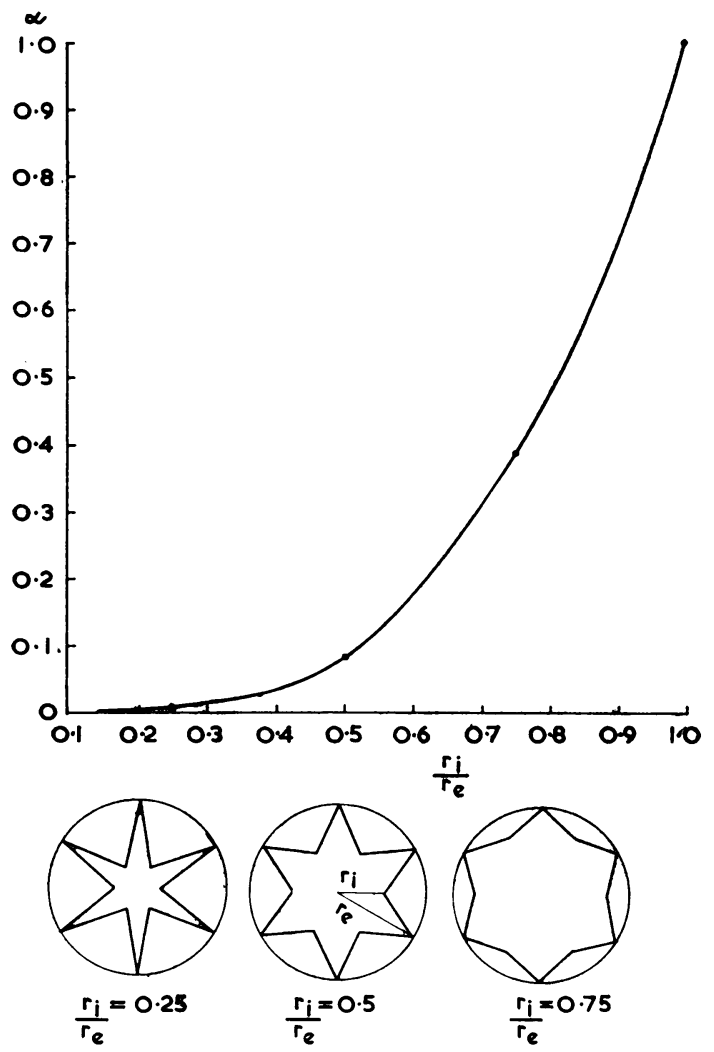

FIG. 2. Hydraulic capacity of a star-shaped pipe $Q=\alpha Q p$ where $Q$ is actual flow and $Q p$ is given by $Q p=\frac{\triangle p}{\triangle 1} \frac{\pi r_{\mathrm{e}}}{8 \mu}$ (Poiseuille flow for a circular pipe of radius $r_{\mathrm{e}}$ ) and $\alpha$ is the coefficient for a star-shaped pipe. Graph of a against $\frac{r_{i}}{r_{\mathrm{e}}}$ where $r_{i}$ is the internal radius and $r_{\mathrm{e}}$ is the external radius. For example, when $\frac{r_{i}}{r_{e}}=$ $0.25, a=0.006$ and hence $Q=0.006 Q p-a$ reduction of flow to $0.6 \%$ and when $\frac{r_{i}}{r_{\mathrm{e}}}=0.75, \alpha=0.384$ and hence $Q=0.384 Q p$-a reduction of flow to $38.4 \%$.

The more closely the star shape approximates to that of a cylinder the more nearly $a=1$. The deeper the indentations into the lumen the more closely does a approach 0. This illustrates the 'choke effect' of a star-shaped tube.

effaced and the lumen approximates to a cylinder, so that at this stage it seems that the hydraulic capacity will tend to follow the unmodified flow equation. Thus, the vestibule permits a large flow through it when fully open.

Clearly the foregoing represents a stylized view of the function of the vestibule, but it is none the less useful as a general consideration.
MODE OF OPENING AND CLOSING OF THE VESTIBULE Cinefluorographic studies have shown that the vestibule opens from above downwards (Fig. 3) and flow normally takes place from above downwards. If, following deglutition, the tension of the muscle falls then that part of the vestibule lying $\nRightarrow$ in the thorax (and subjected to the negative $\vec{\circ}$ intrathoracic pressure) will tend to open first. This may account for the significance of part $\vec{\omega}$ of the vestibule lying in the thorax. In patients with hiatal hernia, in whom the vestibule lies entirely in the thorax, it opens as a whole.

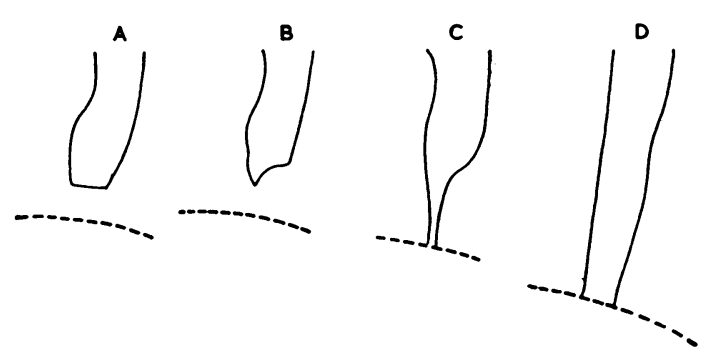

FIG. 3. Tracing from a cine film showing vestibule opening with a non-contractile gullet above. $A$, contrast-filled oesophagus above, closed vestibule, diaphragm shown as a dotted line; $B$ and $C$, successive stages in opening; $D$, vestibule fully open.

As tension in the muscle rises closing of the vestibule must also take place from above downwards, for no contrast medium has been seen to be expelled proximally from the vestibule as it closes.

The above explanation entails an interpretation in terms of both tension and length of muscle (circumference), and manometric studies (Fyke, Code, and Schlegel, 1956) might be thought to support this; but all these studies might also be interpreted solely in terms of length (Harris and Pope, 1964). However, an interpretation in terms of both tension and length is supported by the observations on the function of the vestibule when it lies entirely in the thorax.

RATE OF OPENING AND CLOSING The vestibule opens and closes very rapidly. Measurements have been possible in four patients, and they indicate 0 that the period of time necessary for opening and $\mathbb{D}$ closing is of the order of 0.12 to $0.2 \mathrm{sec}$. This can $\stackrel{?}{+}$ be explained in the following way.

After deglutition the vestibule opens from above downward. When the sum of the forces exerted ( $a$ ) by the fluid column in the gullet above and $(b)$ by the pressures in the thorax and abdomen becomes greater than the falling tension 
exerted by the muscle then the vestibule will open rapidly. It is a characteristic of smooth muscle that it can show 'enormous changes in length with little change in tension' (Woodbury, Gordon, and Conrad, 1965). On closing, the tension in the muscle of the vestibule rises and when it exceeds the sum of the forces exerted $(a)$ by the column of fluid above the vestibule and $(b)$ the negative intrathoracic pressure the vestibule will close and it will do so rapidly.

EFFECT OF DIAPHRAGMaTIC MOVEMENTS During quiet respiration, when the vestibule is open, the calibre of the vestibule is reduced slightly during the inspiratory phase. During expiration the calibre increases slightly. It is possible that this change in calibre may be brought about by the mode of attachment of the vestibule to the diaphragm via the phreno-oesophageal membrane. No localized nipping of the vestibule by the diaphragm was seen. The effects of deep inspiration are complex and have not been analysed in this paper.

\section{DISCUSSION}

Opening of the vestibule is related to deglutition. It is independent of the oesophageal wall and of continuity and patency of the lumen.

Reflux from the stomach through the vestibule into the lower oesophageal pouch has been observed in five patients with oesophageal atresia. By introducing a small volume of contrast medium through a gastrostomy tube and following this by rapidly injecting a quantity of air, gastric distension is produced and the infant behaves as if he were going to vomit. At this time gastro-oesophageal reflux occurs. It is known that rises in pressure in the stomach during vomiting or eructation of gas are not great. Vomiting may also be induced by stimulating the back of the pharynx. These findings suggest that the vestibule can open reflexly as a result of deglutition or with vomiting. In five patients with a lesion of the central nervous system and vomiting free gastrooesophageal reflux was found to be associated with non-closure of the vestibule.

An analysis of the mechanism of opening suggests that following deglutition the tension in the muscle of the vestibule falls. At a critical moment the forces tending to open the vestibule become greater than those tending to close it. When this moment is reached there is a rapid change in circumference of the muscle leading to the development of a lumen within the vestibule. It has been demonstrated that as the vestibule opens a most important influence of the volume of fluid flowing through it is the calibre of the lumen. When the effect of deglutition ceases the tension in the muscle rises and a critical moment is reached when the tension in the muscle is greater than the sum of the forces exerted against it: at this moment the vestibule rapidly closes. These observations and deductions are consistent with the vestibule behaving as a 'muscle throttle' which, when partially open, has the characteristics of a highly efficient choke.

All the findings are consistent with the results of manometric studies published by many workers.

Dornhorst, Harrison, and Pierce (1954) invoked the concept of vestibular function in terms of a valve. This gives the impression that the vestibule functions in a strictly mechanical way, opening and closing being related solely to the pressures in the vicinity. Other authors have also suggested a valvar mechanism-namely, Creamer, Harrison, and Pierce (1959) and Edwards (1962). In infancy and early childhood, when the patient is semi-prone and in the left posterior oblique - projection, it can be seen that on the gastric side of the vestibule a mucosal rosette is produced as the vestibule closes. It seems likely that the rosette is formed because of the way in which the gastric mucosa is loosely attached to the muscle layers of the stomach wall. No mucosal flap, which could in itself close the vestibule, has been identified. The notion of the vestibule behaving as a 'flutter valve' is untenable, for the intraluminal pressure characteristic of the closed vestibule is that it is higher than in the oesophagus above or the stomach below; a flutter valve at its point of closure has a pressure no greater than that immediately outside it. Furthermore, the geometry of the lumen of a flutter valve (Edwards, 1962, see Fig. 35) is not like that of the vestibule. The special circumstances of the patients studied and the way in which it is possible to exclude the thoraco-abdominal pressure difference (see Observations and Deductions, paragraph 2) also indicate that the vestibule does not behave as a flutter valve. Closing of the vestibule from above downward, starting in the thorax, is not consistent with the thoracic pressure being a primary closing mechanism: indeed the negative intrathoracic pressure will tend to keep the vestibule open. The interval between deglutition and opening of the vestibule increases with increasing age, and this is difficult to explain on a purely mechanical basis: so is the finding of an incompetent vestibule in patients with lesions of the 
central nervous system and vomiting. Finally, patients who have had a colon transplant with a direct colon-gastric anastomosis below the diaphragm (as a treatment for severe oesophageal stricture) show free gastro-colonic reflux: the colon provides the flaccid tube of a flutter valve, but no such valvar behaviour is seen. In infants and children the normally located vestibule behaves as a muscle throttle not as a flutter valve.

The vestibule is situated partly in the thorax and partly in the abdomen. The importance of the thoracic location in opening the vestibule has been emphasized. The location of part of the vestibule below the diaphragm is important. In this situation it is subjected to the same extrinsic pressures as the stomach. Thus, rises in intraabdominal pressure are transmitted equally to the stomach and abdominal oesophagus, i.e., the lower part of the vestibule. To prevent gastro-oesophageal reflux only slight tension need be maintained in the muscle of the vestibule to close the mucosal choke.
We wish to thank our clinical colleagues who have referred patients to the Department of Diagnostic Radiology, and in particular Mr. D. J. Waterston, Professor A. W. Wilkinson, and Mr. G. H. MacNab, who were the surgeons concerned with the operative procedures.

\section{REFERENCES}

Chrispin, A. R., and Friedland, G. W. (1966). Proceedings, European Society of Paediatric Radiologists, London Meeting, May 1966.

Creamer, B., Harrison, G. K., and Pierce, J. W. (1959). Further observations on the gastro-oesophageal junction. Thorax, 14, 132.

Dornhorst, A. C., Harrison, G. K., and Pierce, J. W. (1954). Observations on the normal oesophagus and cardia. Lancet, 1, 695.

Edwards, D. A. W. (1962). The "raspberry" or "flutter" valve in the anti-reflux mechanism. In Surgical Physiology of the Gastrointestinal Tract: Proceedings of a Symposium, Royal College of Surgeons of Edinburgh, 1962, ed. A. N. Smith, pp. 24-28.

Fyke, F. E., Code, C. F., and Schlegel, J. F. (1956). The gastroesophageal sphincter in healthy human beings. Gastroenterologia (Basel), 86, 135.

Harris, L. D., and Pope, C. E. (1964). "Squeeze" vs. resistance: An evaluation of the mechanism of sphincter competence. J. clin. evaluation of the
Invest., 43, 2272.

Woodbury, J. W., Gordon, A. M., and Conrad, J. T. (1965). Muscle, In Neurophysiology, by Ruch, T. C., Patton, H. D., Woodbury J. W., and Towe, A. L., 2nd ed., p. 144. Saunders, Philadelphia and London. 